\title{
DESIGN, FORMULATION, AND CHARACTERIZATION OF LIPOSOMAL-ENCAPSULATED GEL FOR TRANSDERMAL DELIVERY OF FLUCONAZOLE
}

\author{
PRINCELY S*, DHANARAJU MD \\ Department of Pharmaceutical Biotechnology, Research Laboratory, GIET School of Pharmacy, Rajahmundry - 533 296, Andhra Pradesh, \\ India. Email: sprincelympharm@gmail.com
}

Received: 03 March 2018, Revised and Accepted: 09 May 2018

\section{ABSTRACT}

Objectives: The present objective for the study was to prepare proliposomal gel bearing an antifungal agent, fluconazole (FLZ) intended for topical application.

Methods: Various proliposome formulations were prepared using thin-film hydration technique by varying the lipid phase composition (phosphatidylcholine/cholesterol). Proliposome formulations were characterized for vesicle size, vesicle size distribution, vesicle morphology, drug content, entrapment efficiency, percentage yield value, storage stability analysis, Fourier-transform infrared spectroscopy (FTIR), differential scanning calorimetry (DSC), in vitro diffusion study, release kinetic studies, and antifungal activity. Topical proliposomal gels were prepared by incorporation of lyophilized proliposome into a structured vehicle carbopol $934(2.5 \%)$

Results: A spherical shape of reconstituted FLZ liposome with an average vesicle about 5-8 $\mu \mathrm{m}$ was observed in photomicrographs. The percentage entrapment of drug was increased with increase in phospholipid composition in the range of 55.13-69.61\%. The FTIR and DSC studies showed no possible drug-excipient interaction. Proliposomal gel showed the prolonged release of FLZ than the lyophilized liposomes. The release kinetic values of regression coefficients confirmed the diffusion-dependent release of the drug. Stability studies indicated that product is stable and should be stored at low temperature.

Conclusion: The proposed FLZ proliposomal gel showed sustained release with enhanced antifungal activity implicating its potential in effective transdermal delivery for the topical pharmacotherapy.

Keywords: Fluconazole (FLZ), Proliposome, Gel, Release kinetics, Transdermal delivery

(c) 2018 The Authors. Published by Innovare Academic Sciences Pvt Ltd. This is an open access article under the CC BY license (http://creativecommons. org/licenses/by/4. 0/) DOI: http://dx.doi.org/10.22159/ajpcr.2018.v11i8.25621

\section{INTRODUCTION}

Liposome is a microparticulate colloidal vesicle, in which aqueous medium is surrounded by single or multiple concentric layers of phospholipids. Due to their size, both hydrophilic and hydrophobic drugs (besides biocompatibility) can be incorporated, water-soluble drug being entrapped in aqueous core and fat-soluble drug in phospholipids [1,2]. It offers controlled release, targeted drug delivery, thus enhancing therapeutic efficacy, and reduced dosing frequency. Therapeutically, these are used as a carrier for drugs, viruses, bacteria, antigen, peptides, antibiotics, vaccines, genes, and diagnostic agents $[3,4]$.

Liposomes are small artificial vesicles of spherical shape that can be created from cholesterol and natural nontoxic phospholipids. Liposome properties differ considerably with lipid composition, surface charge, size, and the method of preparation. Furthermore, the choice of bilayer components determines the "rigidity" or "fluidity" and the charge of the bilayer. For instance, unsaturated phosphatidylcholine (PC) species from natural sources (egg or soybean PC) give much more permeable and less stable bilayers, whereas the saturated phospholipids with long acyl chains (e.g., dipalmitoyl PC) form a rigid, rather impermeable bilayer structure [2].

In general, liposomes are definite as spherical vesicles with particle sizes ranging from $30 \mathrm{~nm}$ to several micrometers. They consist of one or more lipid bilayers surrounding aqueous units, where the polar head groups are oriented in the pathway of the interior and exterior aqueous phases. On the other hand, self-aggregation of polar lipids is not limited to conventional bilayer structures which rely on molecular shape, temperature, and environmental and preparation conditions but may self-assemble into various types of colloidal particles [5]. Liposomes are prepared using sonication, thin-film hydration, solvent dispersion method, and detergent removal methods. Drug loading can be attained either passively (i.e., the drug is encapsulated during liposome formation) or actively (i.e., after liposome formation) [6]

The liposome size can vary from very small $(0.025 \mu \mathrm{m})$ to large (2.5 $\mu \mathrm{m})$ vesicles. Moreover, liposomes may have one or bilayer membranes. The vesicle size is an acute parameter in determining the circulation half-life of liposomes, and both size and number of bilayers affect the amount of drug encapsulation in the liposomes. Liposomes can also be classified into one of two categories: (1) Multilamellar vesicles (MLV) and (2) unilamellar vesicles. Unilamellar vesicles can also be classified into two categories: (1) Large unilamellar vesicles and (2) small unilamellar vesicles. In unilamellar liposomes, the vesicle has a single phospholipid bilayer sphere enclosing the aqueous solution. In multilamellar liposomes, vesicles have an onion structure. Classically, several unilamellar vesicles will form on the inside of the other with smaller size, making a multilamellar structure of concentric phospholipid spheres separated by layers of water [7-9].

Liposomes are found to be suitable for localization of topically applied drugs at or near the site of application because they may act as slowreleasing vehicles. Topical drug delivery is a pleasing route for local and systemic treatment. The delivery of drug through topical route is the most effective treatment for the skin diseases [10]. Finally, liposomal drugs exhibit reduced toxicities and retain enhanced efficacy compared with free complements. However, based on the pharmaceutical 
applications and available products, liposomes have definitely established their position in modern delivery systems [6].

Fluconazole (FLZ) is a first-generation water-soluble triazole antifungal medication that is administered orally or intravenously. It is used to treat a variety of fungal infections, especially Candida infections of the vagina, mouth, throat, bloodstream, fungal keratitis, tinea infection, and coccidioidal meningitis. It is now available as tablet, capsule, injection, and eye drop formulations. The dosage forms have well-known side effects including nausea, vomiting, diarrhea, headache, and abdominal pain. To reduce the disadvantages, the topical gel formulation has been proposed [11].

Agel is a two-component, crosslinked three-dimensional network consisting of structural materials interspersed by an adequate but proportionally large amount of liquid to form an infinite rigid network structure, which immobilizes the liquid continuous phase within [12]. Both hydrophilic and lipophilic drugs can be easily encapsulated in liposomal formulation, and dispensing in the form of carbopol gel was found to be well suited and sound approach to obtain stable liposomal formulation [13]

In the present study, FLZ-loaded multilamellar liposomes were prepared using the thin-film hydration method. The characterization of the prepared liposomes such as vesicle size, vesicle size distribution, zeta potential, vesicle morphology, drug content, entrapment efficiency, percentage yield value, storage stability analysis, Fourier transform infrared spectroscopy (FTIR), and differential scanning colorimetry (DSC) for phospholipid drug compatibility, in vitro diffusion study, release kinetic studies, and antifungal activity was investigated. The selected batch was successfully incorporated into the carbopol to obtain a gel formulation and evaluated for $\mathrm{pH}$ value, conductivity, viscosity, spreadability, and stability. The topical delivery with gels can increase the resistance time of the drug on the skin and improve the delivery and release of the substance by increasing the residence time leading to faster healing to external lesions and to a reduction of side effects and duration of therapy. The purpose of this research was to formulate an effective, stable topical gel containing FLZ with anticipated in vitro characteristics.

\section{MATERIALS AND METHODS}

\section{Materials}

FLZ was obtained from Glaxo Smith Kline Ltd., Mumbai, India. Cholesterol and PC were purchased from Yarrow Chem Products, Mumbai. Methanol, chloroform, and potassium dihydrogen orthophosphate were obtained from Durga Chemicals, Hyderabad, Leo Chem, India, Bengaluru, and Jigchem Universal, Mumbai, respectively. Sodium hydroxide and dimethyl sulfoxide purchased from Paari Chem Resources, Mumbai. Tween-80 was obtained from Sig Chem Products, Mumbai. Carbopol 934 was obtained from Maruti Chemicals, Ahmedabad. Agar, peptone, and dextrose were procured from Marine Chemicals, Kochi, Jeevan chemicals, Mumbai, and Titan Biotech Ltd., Mumbai, respectively. Dialysis tubing membrane procured from Sigma-Aldrich, Mumbai, and all other chemicals used were of pure and analytical grade.

\section{Methods}

\section{Preparation of liposomes with FLZ}

Liposomes were prepared by the method described by Berginc et al., 2014. In brief, FLZ, PC, and cholesterol were dissolved in chloroform:methanol mixture $(2: 1)$ in a round bottom flask. The solvent was evaporated using rotoevaporator system for at least $1 \mathrm{~h}$ at 50 $\mathrm{mm} \mathrm{Hg}$ and $40^{\circ} \mathrm{C}$. The remaining film was then resuspended in $25 \mathrm{~mL}$ of aqueous phase containing $0.25 \mathrm{ml}$ Tween-80 in a homogenizer [14].

\section{Characterization of liposomes}

\section{Vesicle size reduction}

Liposomes were transferred to a beaker and placed on ice bath. The needle probe tip of probe sonicator was placed in the center of the beaker containing liposomal suspension. The sonicator was set to $40 \%$ amplitude, and the liposomes were exposed to ultrasonic irradiation at $37^{\circ} \mathrm{C}(15 \mathrm{~min})$. The sonicated liposomes were stored in the refrigerator $\left(4-8^{\circ} \mathrm{C}\right)$ for at least $6 \mathrm{~h}$ before the further use [15].

\section{Surface morphology (SEM)}

After gold coating of proliposome, their SEM was viewed and photographed by scanning electron microscope [15]

\section{Particle size analysis}

300 globules of prepared liposome of each batch were subjected to determination of vesicle size by optical microscopy method using an eyepiece micrometer calibrated with a stage micrometer. Using the frequency data, the frequency distribution curve was plotted with the average size range on the $\mathrm{x}$-axis and percentage frequency on the $\mathrm{y}$-axis, and the geometrical mean diameter was calculated $[16,17]$.

\section{Lyophilization}

The cryoprotectant glucose ( $1 \mathrm{ml}$ liposomes with $3 \mathrm{~g}$ sucrose) was used with the liposomal formulations and was frozen overnight at $-80^{\circ} \mathrm{C}$ and then freeze-dried for $24 \mathrm{~h}$ under vacuum at $-50^{\circ} \mathrm{C}$. The resulting lyophilized cakes were rehydrated to its original volume at room temperature with PBS and subjected to further investigation. Lyophilized liposomes and non-lyophilized liposomes were kept for stability testing at $4^{\circ} \mathrm{C}, 25 \pm 2^{\circ} \mathrm{C}$, and $40^{\circ} \mathrm{C}$ for 3 months. The percentage drug leakage study was carried out for lyophilized and non-lyophilized liposomes [18,19].

\section{Determination of drug content}

Drug entrapped multilamellar liposomes $(100 \mathrm{mg})$ were suspended in $100 \mathrm{ml}$ solution of chloroform: methanol (2:1). The resultant dispersion was kept for $20 \mathrm{~min}$ for complete mixing with continuous agitation and filtered through a $0.45 \mu \mathrm{m}$ membrane filter. The drug content was determined spectrophotometrically at $261 \mathrm{~nm}$ using a regression equation derived from the standard graph. Results were based on triplicate determination [20].

Drug content $=\frac{\text { Sample absorbance }}{\text { Standard absorbance }} \times 100$

\section{Percentage drug entrapment (PDE)}

Drug entrapment efficiency was calculated using centrifugation method. The liposomal suspension of $1 \mathrm{ml}$ was taken and centrifuged at $3500 \mathrm{rpm}$ for $15 \mathrm{~min}$. The sediment obtained from the centrifugation

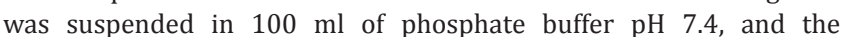
absorbance was taken at $261 \mathrm{~nm}$. From that, the amount present in $1 \mathrm{ml}$ of suspension was obtained. The drug entrapment efficiency was calculated from the following formula [21,22]:

$\%$ Drug Entrapment $(\mathrm{PDE})=\frac{\text { Amount of drug in the sediment }}{\text { Total amount of drug }} \times 100$

\section{Percentage yield of liposomes}

After complete drying, the drug-loaded proliposomes were collected and weighed accurately. The yield of proliposomes was calculated by the following formula [16].

$$
\% \text { Yield }=\frac{\text { Total weight of proliposomes }(\mathrm{g})}{\text { Total weight of drug }+ \text { Total weight of excipents }} \times 100
$$

\section{Compatibility studies}

FTIR

The compatibility between pure drug, cholesterol, and phosphatidylcholine was detected by FTIR analysis. The aim of this study was to test whether there is any interaction between the lipid carriers and drug. Then IR spectrum was recorded individually over the wave number of $8000^{-1}-500 \mathrm{~cm}^{-1}[22]$. 


\section{DSC analysis}

DSC experiments were performed with differential scanning calorimeter (model TA-60, Shimadzu, Japan). Samples of pure FLZ, soya lecithin, cholesterol, and drug-loaded multilamellar liposomes were submitted to DSC analysis. Accurately weighed samples were placed in open, flat bottom, aluminum sample pans. Thermograms were obtained by heating the sample at a constant rate of $10^{\circ} \mathrm{C} / \mathrm{min}$. A dry purge of nitrogen gas $(20 \mathrm{ml} /$ min) was used for all runs. Samples were heated from $35^{\circ} \mathrm{C}$ to $400^{\circ} \mathrm{C}$. Scans were obtained from the samples. The melting point, peak maxima, and the presence and absence of endotherm peaks were observed in the DSC graphs. The temperature of maximal excess heat capacity was defined as the phase transition temperature. DSC analysis was performed to ascertain the lack of potential interactions between the formulations $[23,24]$.

\section{Preparation of liposomal gel}

On the basis of encapsulation efficiency and in vitro drug release, liposome batch F7 (LPE) was selected for further formulation studies of liposomal gel. Gel was prepared using Carbopol 934 (1.5, 2.5, and 3\%) (Moghimipour et al., 2013). The appropriate quantity of Carbopol 934 powder was dispersed into distilled water under constant stirring with a glass rod, taking caution to avoid the development of indispensable lumps, and allowed to hydrate for $24 \mathrm{~h}$ at room temperature for swelling. Topical liposome gel formulations were prepared by incorporation of liposome's containing FLZ and were mixed into the carbopol gel with a mechanical stirrer $(25 \mathrm{rpm}, 2 \mathrm{~min})$. The dispersion was neutralized using triethanolamine $(0.5 \% \mathrm{w} / \mathrm{w})$. Control gels were made under the same conditions $[16,25,26]$.

\section{Physical examination}

\section{Macroscopic analysis of formulation}

The prepared F7-FLZ gel formulation was examined visually for its color, homogeneity, consistency, and spreadability. The clarity was determined using the natural light, and all the macroscopic analyses were analyzed comparing with carbopol [16,25-27].

\section{Conductivity and $p H$ optimization}

The $\mathrm{pH}$ and conductivity of F7-FLZ gel formulation were determined using digital $\mathrm{pH}$ meter. The glass electrode was calibrated with the solutions determined for the equipment ( $\mathrm{pH}$ of 4.00 and 7.00), and the conductivity measurement was done in millivolts. The preparation was left for about 15 min for attaining equilibrium while measuring. The analysis of $\mathrm{pH}$ and conductivity of formulation were done in triplicate, and average values were calculated [16,25-27].

\section{Viscosity study and rheological properties}

The rheological analysis of the experimental gels was performed with a Viscometer (Quimis MOD 0860M21). The gel was rotated at 10, 20, $30,40,50$, and 60 rotations/min. At each speed, the corresponding dial reading was noted. Viscosity of $1 \%, 2 \%$, and $3 \%$ carbopol gel was determined, and optimized concentration was chosen [16,25-27].

\section{In vitro studies}

In vitro release of FLZ from multilamellar liposomes

The release of FLZ from all multilamellar liposomal formulations with different compositions was determined using the membrane diffusion technique. The dialysis tubing membrane (MW cutoff 12000-14000) presoaked overnight in phosphate buffer ( $\mathrm{pH}$ 7.4) was used. Accurately measured amount of FLZ liposomal formulations, equivalent to $20 \mathrm{mg} \mathrm{FLZ,}$ was placed in the dialysis tubing membrane and the ends were sealed. The sealed tubings were placed in a conical flask containing $50 \mathrm{ml}$ phosphate buffer pH 7.4 in an orbital shaker incubator shaken at 100 strokes per minutes. The aliquots of samples were withdrawn from the conical flask at specified intervals up to $24 \mathrm{~h}$ and replaced with same volume of fresh solution. The samples were assayed spectrophotometrically for drug content at $261 \mathrm{~nm}$ [28]. The cumulative drug release was calculated with the help of standard calibration curve of FLZ. The experiments were performed in triplicates, and the results were represented as the mean values of three runs. The obtained results were subjected to kinetic treatment to determine the order of release pattern and release rate constant [29-32].

Optimized gel base (F7) was evaluated for the cumulative drug release using the following method for the gel loaded with liposomes.

\section{In vitro antifungal activity}

The vesicular formulations were assayed for antifungal activity against the fungal strain Candida albicans, obtained from Microbial Type Culture Collection, GSL Medical College, Rajahmundry. Then, $0.1 \mathrm{ml}$ fungal culture suspension was mixed in $9.9 \mathrm{ml}$ Sabouraud's dextrose broth and was incubated for $24 \mathrm{~h}$ at $25^{\circ} \mathrm{C}$. To the sterile Petridishes in which

Table 1: Formulation of FLZ liposomes by thin-film hydration technique

\begin{tabular}{|c|c|c|c|c|c|c|}
\hline Formulation code & FLZ (mg) & Phosphatidylcholine (mg) & Cholesterol (mg) & Chloroform (ml) & Methanol (ml) & Tween-80 (ml) \\
\hline $\mathrm{F} 1$ & 100 & 100 & 10 & 10 & 5 & 0.25 \\
\hline $\mathrm{F} 2$ & 100 & 100 & 30 & 10 & 5 & 0.25 \\
\hline F3 & 100 & 100 & 50 & 10 & 5 & 0.25 \\
\hline F4 & 100 & 150 & 10 & 10 & 5 & 0.25 \\
\hline F5 & 100 & 150 & 30 & 10 & 5 & 0.25 \\
\hline F6 & 100 & 150 & 50 & 10 & 5 & 0.25 \\
\hline F7 & 100 & 200 & 10 & 10 & 5 & 0.25 \\
\hline F9 & 100 & 200 & 50 & 10 & 5 & 0.25 \\
\hline
\end{tabular}

FLZ: Fluconazole

Table 2: Determination of particle size, $\%$ yield, $\%$ drug content, and entrapment efficiency of FLZ liposomes

\begin{tabular}{|c|c|c|c|c|c|c|}
\hline Formulation code & Particle size $(\mu \mathrm{m})$ & Polydispersity index & Swelling index & Percentage yield & $\begin{array}{l}\text { Drug } \\
\text { content (\%) }\end{array}$ & $\begin{array}{l}\text { Entrapment } \\
\text { efficiency (\%) }\end{array}$ \\
\hline F1 & $5.86 \pm 0.29$ & $0.323 \pm 0.03$ & $2.84 \pm 0.37$ & $81.01 \pm 1.52$ & $99.54 \pm 0.81$ & $55.13 \pm 3.12$ \\
\hline $\mathrm{F} 2$ & $6.38 \pm 0.46$ & $0.265 \pm 0.01$ & $2.45 \pm 0.62$ & $84.52 \pm 0.98$ & $98.61 \pm 1.15$ & $56.57 \pm 1.35$ \\
\hline F3 & $6.94 \pm 0.35$ & $0.192 \pm 0.02$ & $2.32 \pm 0.25$ & $86.43 \pm 2.43$ & $98.32 \pm 0.97$ & $58.11 \pm 0.97$ \\
\hline F4 & $6.52 \pm 0.87$ & $0.418 \pm 0.11$ & $2.93 \pm 0.55$ & $75.64 \pm 1.35$ & $97.35 \pm 1.69$ & $61.92 \pm 2.61$ \\
\hline F5 & $6.91 \pm 0.56$ & $0.351 \pm 0.05$ & $3.05 \pm 0.86$ & $77.45 \pm 2.21$ & $96.53 \pm 0.85$ & $63.83 \pm 1.12$ \\
\hline F6 & $7.26 \pm 0.39$ & $0.264 \pm 0.03$ & $3.14 \pm 0.47$ & $79.31 \pm 0.96$ & $96.25 \pm 1.53$ & $65.55 \pm 0.93$ \\
\hline F8 & $8.32 \pm 0.73$ & $0.463 \pm 0.09$ & $3.57 \pm 0.53$ & $73.61 \pm 1.22$ & $94.55 \pm 1.47$ & $67.83 \pm 1.47$ \\
\hline F9 & $8.96 \pm 0.45$ & $0.375 \pm 0.06$ & $3.23 \pm 0.66$ & $74.82 \pm 1.18$ & $94.31 \pm 0.89$ & $69.61 \pm 0.99$ \\
\hline
\end{tabular}

All values are represented as mean \pm standard deviation $(n=3)$, SEM: Standard error mean $(<0.5)$. FLZ: Fluconazole 



Fig. 1: Scanning electron microscopy of the optimized F7 fluconazole and F7 gel liposomes

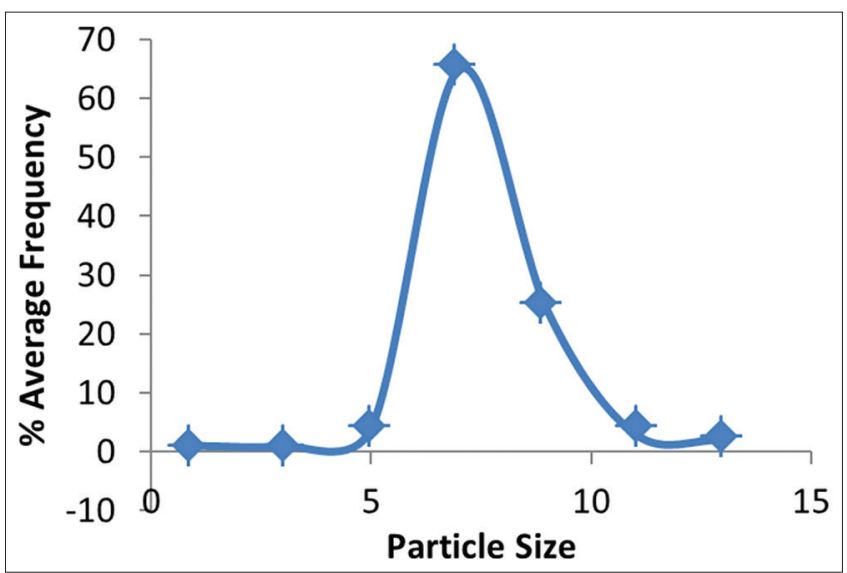

Fig. 2: Particle size frequency distribution profile of the optimized formulation F7

solidified Sabouraud's dextrose broth agar medium were taken, add $1 \mathrm{ml}$ of Sabouraud's dextrose broth containing fungal culture suspension. The inoculum was spread uniformly over the solid agar surface by rotating the plate in clockwise and anticlockwise direction, and three wells were made in the plates with the help of sterile cork borer. The FLZ liposomal formulation (F7, $100 \mu \mathrm{g} / \mathrm{ml}$ ), positive control (pure drug in dimethyl sulfoxide, $100 \mu \mathrm{g} / \mathrm{ml}$ ), and negative control (dimethyl sulfoxide, $100 \mu \mathrm{g} / \mathrm{ml}$ ) were added to the wells and then incubate the plates at $25^{\circ} \mathrm{C}$ for $48 \mathrm{~h}$. The clear rings, i.e., zone of inhibition will appear around the wells. The larger the zone of inhibition, the more effectively the formulation works.

The antifungal activity was evaluated by measuring zones of inhibition of fungal growth surrounding the formulations with scale in $\mathrm{mm}$. The complete antifungal analysis was carried out under strict aseptic conditions. The experiment was carried out in triplicates [33].

\section{Storage stability analysis}

Physical stability study was performed to investigate the leak out of the drug from liposomes during storage. Liposomal suspension of FLZ of optimized batch (F7) was sealed in 20 -ml glass vials and stored at refrigeration temperature $\left(4^{\circ} \mathrm{C}\right.$ and $\left.25^{\circ} \mathrm{C}\right)$ and room temperature $\left(37^{\circ} \mathrm{C}\right.$ and $\left.45^{\circ} \mathrm{C}\right)$ for a period of 45 days. Samples from each liposomal formulation were withdrawn at definite time intervals. The residual amount of the drug in the vesicles was determined after separation from unentrapped drug as described previously under the section drug entrapment efficiency [25,31-34].

The optimized gel base (F7) was evaluated for the following parameters for both plain gel and gel loaded with liposomes. It is tested for changes in SEM, color, and drug content.

\section{Statistical analysis}

The formulations were tested in triplicate, and each analysis was duplicated and expressed as mean+standard error of mean. $p<0.05$ was considered statistically significant.

\section{RESULTS AND DISCUSSION}

\section{Characterization of liposomes}

The proliposomes of FLZ were prepared by thin-film hydration method using Tween-80 which was found to be suitable for the production of liposomes without aggregation. The rationale of the present study was to form MLV to increase permeation of drug, improve therapeutic response, and reduce the possible adverse effects.

In the present investigation, nine formulations using different concentrations of FLZ, PC, cholesterol, chloroform, methanol, Tween 80, and water were prepared using thin-film hydration technique (Table 1). The solubility of FLZ in $10 \mathrm{mg} / 10 \mathrm{ml}$ of solvent was carried out, and it reveals that it is soluble in chloroform: methanol (2:1).

\section{Drug content, SEM, and particle vesicle size}

A standard calibration curve for FLZ was obtained by measuring absorbance at $261 \mathrm{~nm}$ by plotting the graph of absorbance versus concentration between 5 and $30 \mu \mathrm{g} / \mathrm{ml}$ ranges. The particle vesicle sizes of all formulations were found to be in the range between $5.86 \mu \mathrm{m}$ and $8.96 \mu \mathrm{m}$ (Fig 2 ). This method was effective to produce liposome population having narrow size distribution. The results showed that, with an increase in the concentration of phospholipid and cholesterol, the vesicle size was found to be increased (Table 2). The SEM photomicrographs of the optimized formulation F7 reveal the presence of well-identified spheres of MLV that consists of concentric phospholipid bilayers (Fig. 1). In this study, polydispersity index of $<0.537$ indicates that the narrow size distribution of liposome were obtained.

Drug entrapment efficiency

The PDE was found to be in the range of 55.13-69.61\% (Table 2). The study showed that there is an improvement in PDE when there is influence of cholesterol (less molar ratio of PC and cholesterol), but further increase in cholesterol content decreased the PDE.

\section{Percentage yield}

The percentage yield for the liposome formulations was found to be in the range of $71.55-86.43 \%$ and increased with increase in the molar ratio of PC and cholesterol (Table 2). The percentage drug content was found to be maximum for F7 FLZ liposomes (95.64\%) as given in Table 2. It was found that homogenization and sonication had no effect on drug content, whereas phospholipids to drug concentration attributes to the drug content of the formulation.

\section{FTIR and DSC}

On comparison of IR spectra of liposomes, pure drug, PC, and cholesterol, it was clear that there are no possible interactions between FLZ and the other ingredients within the formulation (Fig 3). The DSC results of liposomes suggest enhanced entrapment efficiency of FLZ in the lipid bilayer (Fig 4).

Based on the encapsulation efficiency and in vitro drug release, LPE was selected further for the formulation studies of liposomal gel. Gel was prepared using Carbopol $934(1.5,2.5$, and 3\%) as both plain gel and gel loaded with liposomes.

\section{Characteristics of liposomal gel Macroscopic characteristics}

The F7-FLZ Gel formulations were analyzed for its macroscopic characteristics and qualities such as color, homogeneity, phase separation, and consistency. The gel has a smooth texture, white color, transparent, and characteristic odor of gel extract. The formulations were found to be homogeneous without color intensity, differences, and aggregates.

\section{$p H$}

The $\mathrm{pH}$ values of F7-FLZ Gel were found to be in the range from $3.88 \pm 0.02$ to $3.73 \pm 0.0$, which was expected since the carbopol was 




Fig. 3: Fourier-transform infrared spectroscopy photograph of F7 liposomal formulation



Fig. 4: Differential scanning colorimetry analysis of pure drug, phosphatidylcholine, cholesterol, and F7 liposomal formulation. FLZ: Fluconazole; CHOL: Cholesterol; PC: Phosphatidylcholine; F7: Formulation 7

formulated with $\mathrm{pH}$ between 5 and 5.5 because these are the values sufficient to obtain a good viscosity and clarity of the gel. This $\mathrm{pH}$ value showed that F7-FLZ gel probably would not produce skin irritation. The conductivity values of F7-FLZ gel remained stable. Hence, the prepared F7-FLZ gel is suitable for topical application.

\section{Spreadability and viscosity}

The spreadability of F7-FLZ gel was measured high by having a low spread of time. The therapeutic efficacy of gels depends on their spread.
The gel spreading helps in the uniform application of the gel to the skin, so the prepared gels must have a good spreadability and gratify the ideal quality in topical application. Moreover, this is considered an essential factor in patient compliance with treatment. The uniformity of the substance is one of the most crucial features to topical formulations due to being applied to the thin layers of the skin so that the gel viscosity plays a vital role in regulating drug permeation. The viscosity measured for optimized plain gel and liposomal gel showed 1070 and 1309 centipoises, respectively. The viscosity increases on increasing the polymer concentration. 


\section{Rheological studies}

In rheological study, the semisolid preparations should flow or deform after applying the force and regain its elasticity as the force is removed. Thus, to understand the rheological properties of liposomal gels and for the selection of optimum concentration of carbopol having desired rheological properties, different concentrations $(1.5,2.5,3 \% \mathrm{w} / \mathrm{w})$ of Carbopol 934 were used to prepare liposomal gels at $25^{\circ} \mathrm{C}$ with neutralization method. The rheologies of all samples were determined to identify the minimum concentration of carbopol required for the formation of gel with good viscoelastic properties.

\section{In vitro release study}

Drug release profiles from multilamellar liposomes showed that approximately $10-25 \%$ of the drug is released at a relatively rapid rate during the first $2 \mathrm{~h}$, followed by slower release rates over the next $24 \mathrm{~h}$ (Fig. 5). Hence, doubling the cholesterol ratio decreased the release rate.

The ability of liposomes to retain FLZ during in vitro diffusion studies was also correlated with entrapment values, which indicates that F7 shows sustained drug release, i.e., $66.12 \%$ with smaller vesicle



Fig. 5: Comparative diffusion study profile of fluconazole liposomes size $7.54 \mu \mathrm{m}$, maximum entrapment efficiency $69.61 \%$, and F7-FLZ gel shows sustained drug release of 69.43 . This indicates that the concentration of the polymer has an influence on the drug release. The drug release was decreased with an increase in gelling agent because the polymer concentration increases plus viscosity increases.

\section{Release kinetics}

The kinetic analysis of the release data was carried out by plotting a graph between cumulative drug releases and square root of time. It suggested a linear correlation of drug release with time. The mathematical evaluation of order of release correlation regression coefficient (from the graph) revealed that the drug release followed zero order>first order $>$ Higuchi model by the regression values of the respective plots. The release kinetics for $\mathrm{F} 7$ gel drug release followed first order $>$ zero order $>$ Higuchi model by the regression values (Fig. 6). The values of regression coefficients confirmed the diffusion-dependent release of the drug. The release data were fitted with different kinetic equations to ascertain the drug release mechanism from the gel matrix.

In vitro antifungal study showed all formulations with varying degree of the zone of inhibition area on $1^{\text {st }}$ day, $2^{\text {nd }}$ day, and $3^{\text {rd }}$ day when measured (Fig. 7). This may be due to the difference in the rate of drug release from vesicular carriers.

\section{Stability studies}

In storage stability analysis, acceleration in drug leakage and increase in vesicle size at higher temperatures suggested keeping the liposomal product in the refrigeration condition. F7 liposomes were found to be reasonably stable in terms of aggregation, vesicle disruption, and fusion over the studied storage period (Fig. 8). It can be concluded that, at room temperature and freeze temperature, there was slightly but insignificantly decrease in PDE and increase in particle size for F7 batch. The results suggest that lyophilized or refrigerated liposomal products minimize stability problems of liposomes.

By comparing with the previous reports of FLZ nanocapsules (de Assis et al., 2008), FLZ multilamellar liposomes (El-Nesr et al., 2010), FLZ liposomes (Chanda et al., 2011), FLZ topical gel (Das et al., 2014), FLZ proniosomal gel (Sandeep et al., 2014), and in vitro characterization of ketoconazole liposomal gel (Prakash et al., 2017) there was better

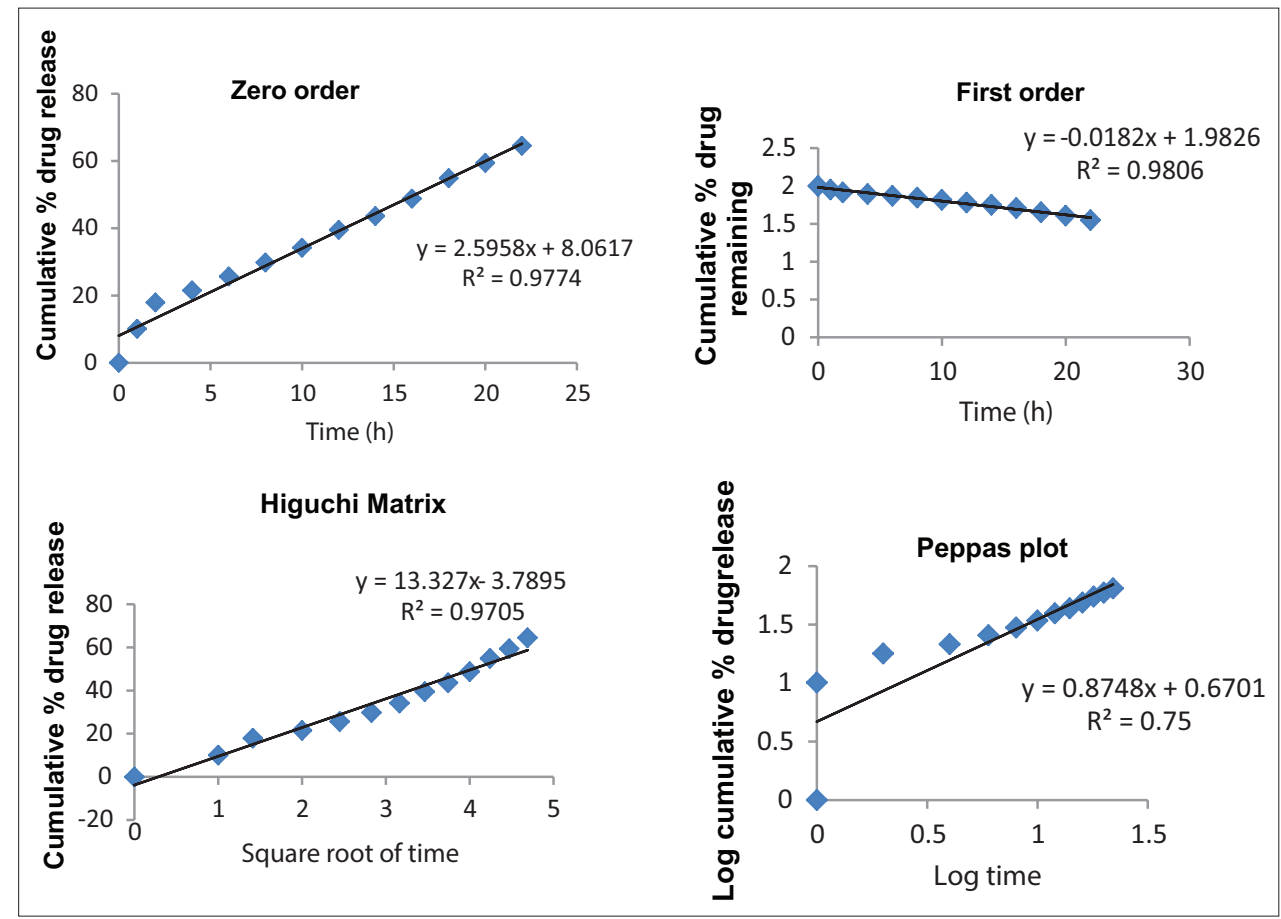

Fig. 6: Kinetic equations for optimized formulation F7 FLZ gel liposomes 

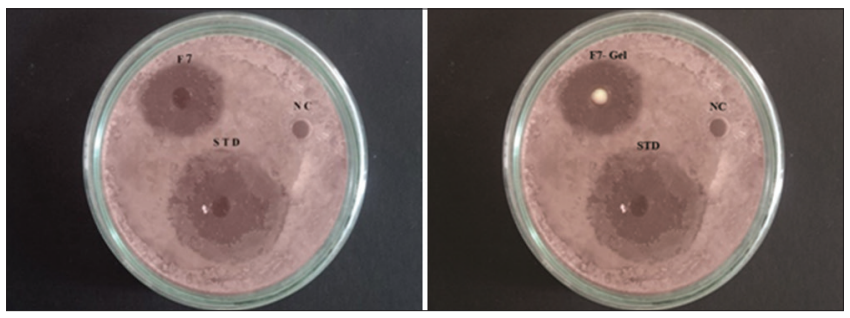

Fig. 7: Zone of inhibition of optimized formulation F7 and F7 gel against Candida albicans

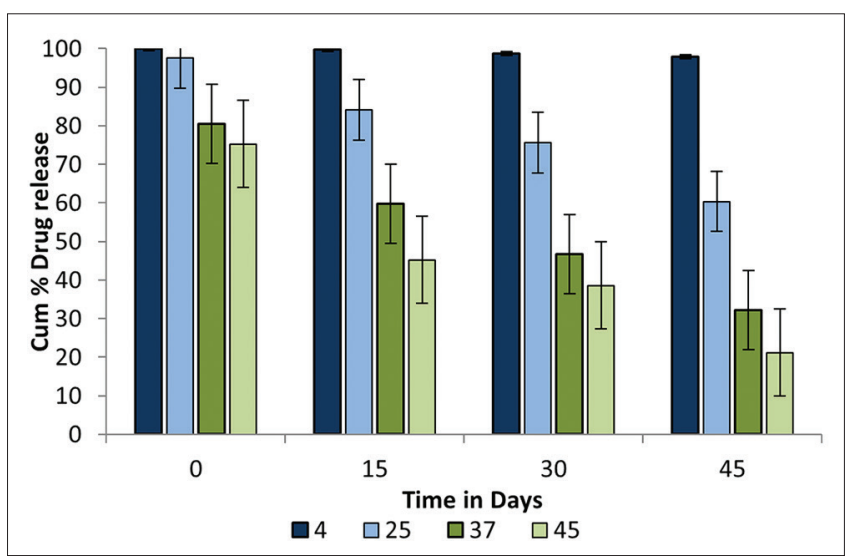

Fig. 8: Percentage of drug remaining in liposomes and liposomal gel under different storage conditions at $4^{\circ} \mathrm{C}, 25^{\circ} \mathrm{C}, 37^{\circ} \mathrm{C}$, and $45^{\circ} \mathrm{C}$

effective controlled release observed in this investigation. It is evident that promising controlled release liposomes can be developed by film hydration technique using PC, cholesterol, chloroform, methanol, and Tween-80.

The selected F7-FLZ was successfully incorporated into the carbopol to obtain a gel formulation. The F7 gel presented good pH value, conductivity, viscosity, spreadability, and stability. Rheological studies of all liposomal gels prepared with carbopol provided a clear knowledge of concentration of carbopol required, i.e. $2.5 \% \mathrm{w} / \mathrm{w}$ Carbopol 934 . Stability studies performed for F7 liposomal dispersion and F7-gel indicate that the prepared liposomes have more stability at freezing temperature than that of room temperature. FLZ molecules could be effectively entrapped in liposomes with reasonable drug loading. This preliminary study should be continued further to learn the impact of polymer concentrations and survey of other cryoprotectants. The optimization of drug release to acquire an effective delivery system could be investigated on animal models for progressive exploration in the near future.

\section{CONCLUSION}

The FLZ-entrapped multilamellar liposomes were successfully prepared using thin-film hydration technique with the view to improve therapeutic response, enhance retention of drug molecule in the skin, and reduce the possible adverse symptoms. The prepared liposomes were found to have good morphological properties and size distribution influenced by bilayer cholesterol content. The percentage entrapment efficiency was optimized after studying the effect of various process and formulation variables. The incorporation of cholesterol increased the rigidity of the bilayer and lowered the permeability of FLZ from liposomes, resulting in greater drug retention. Variables such as amount of phospholipid and amount of stabilizer have an intense effect on the vesicle size and entrapment efficiency. Hence, from results obtained, it can be concluded that liposomal gel containing FLZ could be very promising alternative for the topical or transdermal treatment. However, further preclinical, clinical, and long-term stability studies should be required.

\section{AUTHOR'S CONTRIBUTION}

The author verified the analytical methods, encouraged to investigate formulations for successful topical drug delivery approaches, and supervised the findings of this work.

\section{CONFLICTS OF INTEREST}

There are no conflicts of interest to declare.

\section{REFERENCES}

1. Abolfazl A, Rogaie RS, Soodabeh D, Sang WJ, Nosratollah Z, Younes H, et al. Liposome: Classification, preparation, and applications. Nanoscale Res Lett 2013;8:102.

2. Agnieszka EW, Emilian A. Interfacial properties of phosphatidylcholinebased dispersed systems. Ind Eng Chem Res 2015;54:6489-96.

3. Zhenjun H, Xuan L, Ting Z, Yanzhi S, Zhennan S, Jing L, et al. Progress involving new techniques for liposome preparation. Asian J Pharm Sci 2014;9:176-82.

4. Maximiliano G, Claudia S, Carlos B, Adriana MC. Percutaneous drug delivery systems for improving antifungal therapy effectiveness: A review. Int J Pharm Pharm Sci 2014;6:8-16.

5. Loveleenpreet K, Prabhjot K, Khan MU. Liposome as a drug carrier-A review. Int J Res Pharm Chem 2013;3:121-8.

6. Kamra M, Diwan A. Liposomes in dermatological diseases. J Appl Pharm Res 2017;5:1-8.

7. Bhupendra P, Narendra K, Suman S, Amit R. Liposome: Method of preparation, advantages, evaluation and its application. J Appl Pharm Res 2015;3:1-8.

8. Gyati S, Davinder S, Abhay A. Novel vesicular carriers for topical drug delivery and their applications. Int J Pharm Sci Rev Res 2013;21:77-86.

9. Marilene E, Maria HA, Jaime C, José MS. Evolution of liposomal carriers intended to anticancer drug delivery: An overview. Int J Curr Pharm Res 2015;7:26-33.

10. Prasanthi D, Lakshmi PK. Vesicles-mechanism of transdermal permeation: A Review. Asian J Pharm Clin Res 2012;5:18-25.

11. Sudipta D, Arnab S, Ananya B. Design, development and evaluation of fluconazole topical gel. Asian J Pharm Clin Res 2015;8:132-5.

12. Gagan G, Tarun G, Basant M, Gaurav C, Goutam R, Amit KG. Development and characterization of niosomal gel for topical delivery of benzoyl peroxide. Drug Deliv 2016;22:1027-42.

13. Susanta P, Mayukh J, Anannya B, Sunirmal B, Joyeeta GR, Puja C. Topical liposomal gel: A new strategy of novel drug delivery. World J Pharm Pharm Sci 2016;6:568-77.

14. Dheeraj N, Nidhi A, Deepshikha K. Evaluation of liposomal gossypin in animal models of epilepsy. Int J Pharm Pharm Sci 2016;8:247-51

15. Subash C, Pandey VP. Formulation and evaluation of Glimepirideloaded liposomes by ethanol-injection method. Asian J Pharm Clin Res 2016:9:192-5

16. Fatima T, Raza M, Shukla AK. Development and characterization of prednisolone liposomal gel for the treatment of rheumatoid arthritis. Int Res J Pharm 2015:6:133-7.

17. Ahmed AN, Ahmed GH, Amina LM, Marwa MM, Nabil YA. Liposomal Microencapsulation of rodent-repelling agents onto Jute Burlaps: Assessment of Cytotoxicity and rat behavioural test. J Appl Pharm Sci 2016;6:142-50.

18. Olga P, Jana S, Zoran K, Vesna R. An overview: Methods for preparation and characterization of liposomes as drug delivery systems. Int J Pharm Phytopharm Res 2013;3:13-20.

19. Amol AT, Arun TP, Milind JU, Darshan RT. Investigation of effect of phospholipids on physical and functional characterization of Paclitaxel liposomes. Int J Pharm Pharm Sci 2017;9:141-6.

20. Cedar HAB, Ketan P, Mandip S. Doxorubicin liposomes as an investigative model to study the skin permeation of nanocarriers. Int J Pharm 2015;489:1-11

21. Zhengzi Q, Xianhuo W, Zheng S, Huilai Z, Shiyong Z, Jing Z, et al. A Phase I Trial to evaluate the multiple-dose safety and antitumor activity of Ursolic acid liposomes in subjects with advanced solid tumors. Bio Med Res Int 2015;2015:1-7.

22. Srinivas MB, Vishakha K, Prakash P, Jamal BD, Govardhan T, Muni SP. Formulation and in vitro evaluation of liposomes loaded with mupirocin. Int J Res Pharm Nano Sci 2015;4:162-74.

23. Monika B, Szilvia B, Maria BS, Anita K, Erzsebet C. DSC for evaluating the encapsulation efficiency of lidocaine loaded liposomes compared to the ultracentrifugation method. J Therm Anal Calorim 2017;130:1619-25 
24. Eskandar M, Mohsen T, Ali B, Somayeh H. Formulation and in vitro evaluation of topical liposomal gel of triamcinolone acetonide. Res J Pharm Biol Chem Sci 2013;4:101-7.

25. Milla GB, Silvio AG, Camila MD, Larissa AR, Pedro JR, Ferdinando OC, et al. Development and evaluation of stability of a gel formulation containing the Monoterpene Borneol Sci World J 2016;2016:1-4.

26. Panuganti V, Ellutla M, Prasanna RY, Vayalpati AR, Bandugalla SR, Basappa K. Liposomal Aloe vera trans-emulgel drug delivery of naproxen and nimesulide: A study. Int J Pharma Investig 2015;5:28-34.

27. Khalid ME, Fathy IA, Ahmed EL, Abd ES, Alaa EA. Diacereinniosomal gel for topical delivery: Development, in vitro and in vivo assessment. J Lipos Res 2016;26:57-68.

28. Girish S, Seth AK, Ghanshyam P, Sachin C, Ankur J. Formulation and in vitro evaluation of berberine containing liposome optimized by $3^{2}$ full factorial designs. J Appl Pharm Sci 2015;5:23-8.

29. Sharma NK, Kumar V. Release kinetics of novel photosensitive liposome for triggered delivery of entrapped drug. Int J Pharm Tech
Res 2015;8:106-13.

30. Yan L, Ruiyuan L, Jun Y, Yuanjie S, Guanghui M, Zhenzhong Z, et al. Enhanced retention and anti-tumor efficacy of liposomes by changing their cellular uptake and pharmacokinetics behaviour. Biomaterial 2015;41:1-14

31. Shabnam, Prathima S, Ravindra BD. Formulation and evaluation of parenteral Methotrexate nanoliposomes. Int J Pharm Pharm Sci 2014;6:295-300.

32. Akshay SR, Sudipta D, Arnab S. Design, formulation and evaluation of liposome containing isoniazid. Int J Appl Pharm 2018;10:52-6.

33. Meghana G, Narayana RK, Siddhartha VT, Raviteja G, Saikrishna RC, Ganesh GN. Formulation and evaluation of Tolnaftate loaded topical liposomal gel for effective skin drug delivery to treat fungal diseases. J Chem Pharm Res 2014;6:856-66.

34. Eman SE, Amna MM, Abeer MK, Doaa GH. Nanoemulsion gel of nutraceutical co-enzyme Q10 as an alternative to conventional topical delivery system to enhance skin permeability and antiwrinkle efficiency. Int J Pharm Pharm Sci 2017;9:207-17. 